\title{
Abbreviations and Identifying Titles
}

\begin{tabular}{|c|c|}
\hline $\mathrm{AC}(\mathrm{O})$ & Assistant Controller Overseas \\
\hline $\mathrm{AC}(\mathrm{P})$ & Assistant Controller Programmes \\
\hline AEF & American Expeditionary Force \\
\hline ASNE HS & Assistant Senior News Editor Home Service \\
\hline BBC NI & BBC Northern Ireland \\
\hline BBCWAC & BBC Written Archives Center \\
\hline $\mathrm{C}(\mathrm{H})$ & Controller Home \\
\hline $\mathrm{CAB}$ & Cabinet Papers \\
\hline $\mathrm{CBB}$ & Cahal Brugha Barracks \\
\hline $\mathrm{CP}$ & Controller Programmes \\
\hline $\mathrm{D} / \mathrm{T}, \mathrm{S}$ & Department of the Taoiseach, $\mathrm{S}$ files \\
\hline DCCR & Dublin Consulate Classified Records \\
\hline DCGR & Dublin Consulate General Records \\
\hline DDG & Deputy Director General \\
\hline DDP & Deputy Director Programming \\
\hline DFD & Deputy Foreign Director \\
\hline DG & Director General \\
\hline DGFP & Documents on German Foreign Policy \\
\hline DGP & David Gray Papers \\
\hline DLCR & Dublin Legation Classified Records \\
\hline DLGR & Dublin Legation General Records \\
\hline DO & Dominions Office \\
\hline DP & Director Programmes \\
\hline DPP & Director Programme Planning \\
\hline DT & Director Talks \\
\hline $\mathrm{EH}$ & Department of Publicity in Enemy Countries \\
\hline ENTE & Empire News Talks Editors \\
\hline $\mathrm{ESD}$ & Empire Service Director \\
\hline FCCGC & $\begin{array}{l}\text { Federal Communications Commission General } \\
\text { Correspondence }\end{array}$ \\
\hline FDRL & Franklin D. Roosevelt Library \\
\hline FGP & Frank Gallagher Papers \\
\hline FIS & Foreign Information Service \\
\hline $\mathrm{FO}$ & Foreign Office \\
\hline FRUS & Foreign Relations of the United States \\
\hline GIB & Government Information Bureau \\
\hline GPO & Government Printing Office \\
\hline
\end{tabular}




$\begin{array}{ll}\text { HCD } & \text { House of Commons Debates } \\ \text { HHP } & \text { Harry Hopkins Papers } \\ \text { HL } & \text { Houghton Library } \\ \text { HMP } & \text { Henry Morganthau Papers } \\ \text { HO } & \text { Home Office } \\ \text { INL } & \text { Irish National Library } \\ \text { IRA } & \text { Irish Republican Army } \\ \text { ISC } & \text { Ireland Situation Committee } \\ \text { JBP } & \text { John Betjeman Papers } \\ \text { JDP } & \text { James L. O'Donovan Papers } \\ \text { JWP } & \text { John G. Winant Papers } \\ \text { LLGR } & \text { London Legation General Records } \\ \text { MA } & \text { Military Archives, Dublin } \\ \text { MGP } & \text { Joseph P. McGarrity Papers } \\ \text { MOI } & \text { Ministry of Information } \\ \text { MPPDA } & \text { Motion Picture Producers and Distributors of America } \\ \text { NAUS } & \text { National Archives of the United States } \\ \text { OCI } & \text { Office of the Coordinator of Information } \\ \text { OCR } & \text { Office of the Controller of Records } \\ \text { OOB } & \text { Overseas Operations Branch } \\ \text { OPC } & \text { Overseas Propaganda Committee } \\ \text { OSS } & \text { Organization of Strategic Services files } \\ \text { OWI } & \text { Office of War Information } \\ \text { PFOD } & \text { Publicity File Overseas Division } \\ \text { PID } & \text { Political Intelligence Department } \\ \text { PRO } & \text { Public Records Office } \\ \text { PSF } & \text { Presidential Secretary's File } \\ \text { RAD } & \text { Records, American Division } \\ \text { RE } & \text { Radio Eireann } \\ \text { RJH } & \text { Richard J. Hayes Papers } \\ \text { ROD } & \text { Records, Overseas Division } \\ \text { RP } & \text { Recorded Programmes } \\ \text { RSP } & \text { Robert E. Sherwood Papers } \\ \text { S/S } & \text { Secretary of State } \\ \text { SPO } & \text { State Paper Office } \\ \text { SWP } & \text { Sumner Welles Papers } \\ \text { TCD } & \text { Trinity College, Dublin } \\ \text { UCD } & \text { University College, Dublin } \\ \text { UVL } & \text { University of Victoria Library } \\ \text { WO } & \text { War Office } \\ & \end{array}$

